\title{
Re-evaluation of the TSL for Yttrium Hydride
}

\author{
Michael L. Zerkle ${ }^{1}$, Jesse C. Holmes ${ }^{1}$ and Jonathan L. Wormald ${ }^{2}$ \\ ${ }^{1}$ Naval Nuclear Laboratory \\ PO Box 79, West Mifflin, PA 15122-0079, USA \\ ${ }^{2}$ Naval Nuclear Laboratory \\ PO Box 1072, Schenectady, NY 12301-1072, USA
}

Michael.Zerkle@unnpp.gov, Jesse.Holmes@unnpp.gov, Jonathan.Wormald@unnpp.gov

\begin{abstract}
Yttrium hydride $\left(\mathrm{YH}_{\mathrm{x}}\right)$ is of interest as a high-temperature moderator material in advanced nuclear reactor systems because of its superior ability to retain hydrogen at elevated temperatures. Thermal neutron scattering laws (TSL) for hydrogen bound in yttrium hydride $\left(\mathrm{H}-\mathrm{YH}_{2}\right)$ and yttrium bound in yttrium hydride $\left(\mathrm{Y}^{-} \mathrm{YH}_{2}\right)$ were previously evaluated by Naval Nuclear Laboratory using the ab initio approach and released in ENDF/B-VIII.0. In that work, density functional theory, incorporating the generalized gradient approximation (GGA) for the exchange-correlation energy, was used to simulate the face-centered cubic structure of $\mathrm{YH}_{2}$ and calculate the interatomic Hellmann-Feynman forces for a $2 \times 2 \times 2$ supercell containing 96 atoms. Lattice dynamics calculations using PHONON were used to determine the phonon density of states. The calculated phonon density of states for $\mathrm{H}$ and $\mathrm{Y}$ in $\mathrm{YH}_{2}$ were then used to prepare $\mathrm{H}-\mathrm{YH}_{2}$ and $\mathrm{Y}-\mathrm{YH}_{2}$ TSL evaluations, in the incoherent approximation, using the LEAPR module of NJOY. In addition elastic scattering was assumed to be incoherent for both $\mathrm{H}$ and $\mathrm{Y}$. While the incoherent elastic scattering approximation is appropriate for $\mathrm{H}-\mathrm{YH}_{2}$, it introduces an undesirable approximation for $\mathrm{Y}_{-} \mathrm{YH}_{2}$. In this work, we re-evaluate the TSL for $\mathrm{Y}^{-} \mathrm{YH}_{2}$ using FLASSH (Full Law Analysis Scattering System Hub). $\mathrm{Y}^{-} \mathrm{YH}_{2}$ is evaluated using the FLASSH generalized coherent elastic scattering capability in order to capture the Bragg peaks associated with the $\mathrm{YH}_{2}$ crystal structure which were neglected in the prior NJOY-based evaluation due to limitations in LEAPR. An experimental approach to validate the $\mathrm{Y}^{-} \mathrm{YH}_{2} \mathrm{TSL}$ using neutron transmission measurements is discussed.
\end{abstract}

KEYWORDS: TSL, thermal neutron scattering, yttrium hydride

\section{INTRODUCTION}

Yttrium hydride $\left(\mathrm{YH}_{x}\right)$ is of interest as a high-temperature moderator material in advanced nuclear reactor systems because of its superior ability to retain hydrogen at elevated temperatures. The microstructure of $\mathrm{YH}_{x}$ at $x \sim 1.6$, the yttrium hydride composition range typically considered to be of practical value in nuclear reactor design, consists of a two-phase solid solution of $\alpha$-Yttrium and $\mathrm{YH}_{2}$ phases [1]. The crystal binding effects of hydrogen in $\mathrm{YH}_{2}$ produce narrow, well-separated energy peaks in the neutron scattering cross section of $\mathrm{YH}_{x}$ at thermal energies. This behavior is characteristic of many metal-hydrides and greatly influences neutron thermalization effects. 
In neutron transport calculations, ENDF thermal scattering law sublibraries are used to capture crystal binding effects on low-energy neutron scattering. The TSL, $S(\alpha, \beta)$, is a temperature-dependent probability distribution defining the possible scattering states for an incident neutron. It has contributions from distinct effects (d) due to scattered wave interference between different nuclear scattering sites and self (s) noninterference effects [2].

$$
S(\alpha, \beta)=S_{\mathrm{d}}(\alpha, \beta)+S_{\mathrm{s}}(\alpha, \beta) .
$$

The momentum and energy transfer resulting from scattering are described by the unitless parameters $\alpha$ and $\beta$ defined as,

$$
\begin{gathered}
\alpha=\frac{E-E^{\prime}-\mu \sqrt{E E^{\prime}}}{A k_{\mathrm{B}} T} \\
\beta=\frac{E-E^{\prime}}{k_{\mathrm{B}} T}
\end{gathered}
$$

where $E$ and $E^{\prime}$ are the incident and scattered neutron energies, $\mu$ is the cosine of the scattering angle, $A$ is the nuclide to neutron mass ratio, $k_{\mathrm{B}}$ is the Boltzmann constant, and $T$ is temperature. In crystals, phonon expansion is used to compute the TSL such that $[2,3]$,

$$
S(\alpha, \beta)=\sum_{p} S^{p}(\alpha, \beta)
$$

which is a summation of increasing orders of the convolution of the vibrational (phonon) spectra, with $p=$ 0 representing elastic scattering and $p>0$ representing inelastic scattering. In current ENDF thermal scattering sublibraries, the incoherent approximation is applied such that distinct effects on inelastic scattering are neglected. Within this approximation, the double-differential scattering cross section is related to the TSL using Fermi's Golden rule as [2],

$$
\frac{\partial^{2} \sigma}{\partial \Omega \partial E^{\prime}}=\frac{1}{k_{\mathrm{B}} T} \sqrt{\frac{E^{\prime}}{E}}\left[\sigma_{\mathrm{coh}}\left(S_{\mathrm{d}}^{0}(\alpha, \beta)+S_{\mathrm{s}}^{0}(\alpha, \beta)+\sum_{p>0} S_{\mathrm{s}}^{p}(\alpha, \beta)\right)+\sigma_{\mathrm{inc}} S_{\mathrm{s}}(\alpha, \beta)\right]
$$

where $\sigma_{\mathrm{coh}}$ and $\sigma_{\mathrm{inc}}$ are the bound coherent and incoherent nuclear scattering cross sections of the nuclide.

The ENDF/B-VIII.0 H-YH $\mathrm{H}_{2}$ and $\mathrm{Y}^{-} \mathrm{YH}_{2}$ TSL evaluations [4] were generated in the incoherent approximation using the LEAPR module of NJOY based on vibrational spectra calculated using the $a b$ initio approach [5]. Elastic scattering was assumed to be incoherent for both hydrogen and yttrium, such that $S_{\mathrm{d}}^{0}(\alpha, \beta)=0$. This approximation is reasonable for hydrogen since its nuclear scattering cross section is primarily incoherent. However, this approximation is not appropriate for yttrium since its nuclear scattering cross section is primarily coherent and it neglects the effects of crystal structure important for coherent elastic scattering from yttrium bound in $\mathrm{YH}_{2}$. When developing the ENDF/B-VIII.0 Y-YH evaluation, use of this approximation was driven by the inability of the LEAPR module of NJOY [6] to treat general crystal structures when calculating coherent elastic scattering cross sections. LEAPR is only able to accurately treat certain hard-coded crystal structures.

In this work, we re-evaluate the Y-YH $\mathrm{YH}_{2}$ TSL using the FLASSH (Full Law Analysis Scattering System Hub) code [7]. In particular, $\mathrm{Y}_{-} \mathrm{YH}_{2}$ is re-evaluated using the FLASSH generalized coherent elastic scattering capability [8] in order to capture the Bragg edges associated with the $\mathrm{YH}_{2}$ crystal structure which were neglected in the prior evaluation due to limitations in LEAPR. 


\section{CALCULATION OF PHONON DOS}

$\mathrm{YH}_{2}$ has a $\mathrm{CaF}_{2}$-type face-centered cubic (FCC) structure. The unit cell is composed of 12 atoms with hydrogen atoms (rose) located in the tetrahedral holes between the $\mathrm{Y}$ atoms (blue) as illustrated in Fig. 1. The VASP code $[9,10]$ was used to perform first-principles quantum mechanics simulations of the $\mathrm{YH}_{2}$ lattice structure using density functional theory (DFT). The $\mathrm{YH}_{2}$ lattice structure was optimized using a total electronic energy threshold of $10^{-6} \mathrm{eV}$, a $0.11 \AA^{-1} \mathrm{k}$-point spacing $(19 \times 19 \times 19 \mathrm{k}$-point mesh), a $500 \mathrm{eV}$ planewave cutoff energy, and the GGA-PBE [11] exchange-energy correlation functional. The VASP optimized $0 \mathrm{~K}$ lattice constant of $a=5.2032 \AA$ is in excellent agreement with the experimental value of $5.2032 \pm 0.0003 \AA$ determined by Daou and Vajda [12] at $90 \mathrm{~K}$ using X-ray diffraction measurements.

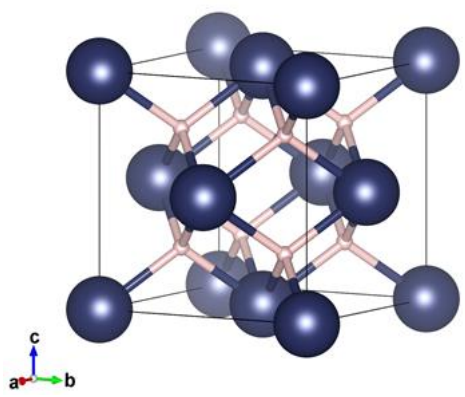

Figure 1. The $\mathrm{YH}_{2}$ Unit Cell.

Lattice dynamics calculations were performed to determine the phonon density of states (DOS) for $\mathrm{YH}_{2}$ using the PHONON [13] code. Interatomic Hellman-Feynman forces on a $2 \times 2 \times 2$ supercell ( 96 atoms) with $\pm 0.02 \AA$ asymmetric atom displacements were determined from a series of VASP calculations using the GGA-PBE pseudopotential, a $500 \mathrm{eV}$ planewave cutoff energy, and a $0.086 \AA^{-1} \mathrm{k}$-point spacing $(7 \times 7 \times 7 \mathrm{k}$ point mesh) and a $0.11 \AA^{-1} \mathrm{k}$-point spacing $(19 \times 19 \times 19 \mathrm{k}$-point mesh) for the electronic energy and DOS contribution to thermodynamic function calculations.

Fig. 2 provides a comparision of the calculated $\mathrm{H}$ and $\mathrm{Y}$ partial phonon DOS for $\mathrm{YH}_{2}[5]$ to the $\mathrm{YH}_{2}$ optical mode phonon DOS measured by Udovic [16]. The phonon DOS has two well-separated regions (due to the large mass ratio between $\mathrm{Y}$ and $\mathrm{H})$ : an acoustical region $(0-0.028 \mathrm{eV})$ which is the preferred region for heavier Y atom vibrations and an optical region $(0.112-0.142 \mathrm{eV})$ which is the preferred region for lighter $\mathrm{H}$ atom vibrations. The optical region is centered at $\sim 0.127 \mathrm{eV}$ which is in agreement with the $0.128 \pm$ $0.004 \mathrm{eV}$ estimate by Flotow [14] derived from heat capacity measurements and the $0.127 \pm 0.007 \mathrm{eV}$ value recommended by Rush [15] based on inelastic neutron scattering measurements. As shown in Fig. 2, the $\mathrm{YH}_{2}$ optical mode from high-resolution inelastic neutron scattering measurements by Udovic [16] has a similar bimodal structure centered at $\sim 0.127 \mathrm{eV}$ and is in excellent agreement with the theoretically calculated phonon DOS. Thermodynamic properties of $\mathrm{YH}_{2}$ can also be calculated from the phonon density of states. Fig. 3 shows the calculated heat capacity for $\mathrm{YH}_{2}$ is in agreement with the measurements by Flotow [14].

\section{THERMAL NEUTRON SCATTERING LAW}

The $\mathrm{Y}-\mathrm{YH}_{2}$ TSL $(\mathrm{MAT}=55)$ was re-evaluated at 10 temperatures between 293.6 and $1600 \mathrm{~K}$ using the FLASSH code [7]. The generalized coherent elastic scattering module [8] was used to calculate the elastic scattering cross sections $(\mathrm{MT}=2)$ in the cubic approximation using the $\mathrm{FCC}$ crystal structure of $\mathrm{YH}_{2}$, the room temperature $\mathrm{YH}_{2}$ lattice parameter of $a=5.2085 \AA$ measured by Daou and Vajda [12], and a ${ }^{89} \mathrm{Y}$ freeatom scattering cross section of $7.688246 \mathrm{~b}$ to ensure consistency with the ENDF/B-VIII.0 ${ }^{89} \mathrm{Y}$ evaluation. 


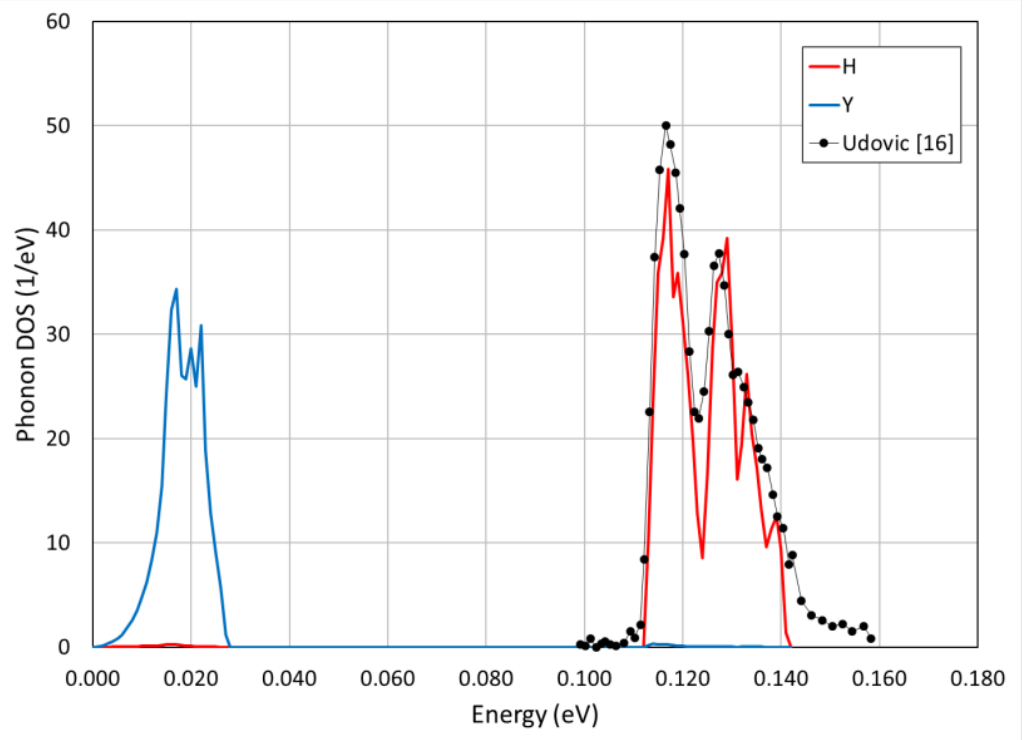

Figure 2. Comparison of Calculated [5] and Measured [16] Phonon DOS for $\mathrm{YH}_{2}$

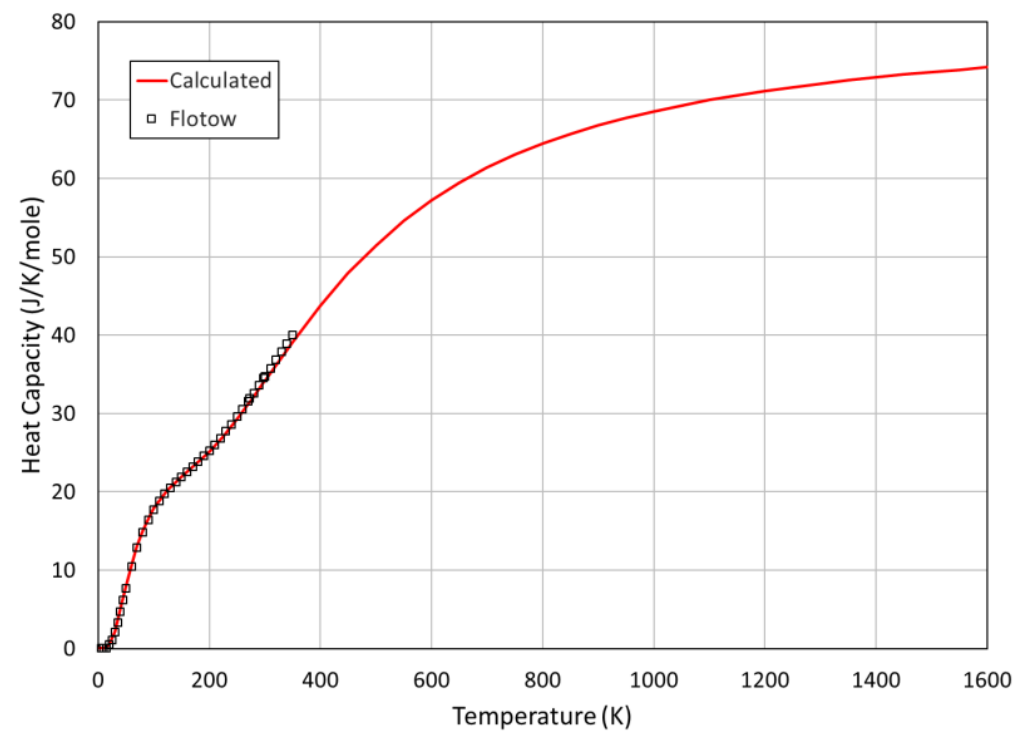

Figure 3. Calculated Molar Heat Capacity for $\mathrm{YH}_{2}$

Use of the cubic approximation is appropriate since $\mathrm{YH}_{2}$ has a FCC crystal structure. The inelastic scattering cross sections $(\mathrm{MT}=4)$ were calculated in the incoherent approximation using the phonon DOS for $\mathrm{Y}$ in $\mathrm{YH}_{2}$ from Fig. 2 (this is the same phonon DOS used to prepare the ENDF/B-VIII.0 Y-YH evaluation) and a phonon expansion order of 300. Fig. 4 provides the coherent elastic scattering and inelastic scattering cross sections calculated by FLASSH. Bragg peaks characteristic of the FCC crystal structure are clearly evident in the elastic scattering cross sections and they behave as expected with increasing temperature. In contrast, the inelastic scattering cross section increases in magnitude with temperature and demonstrates the typical $1 / v$ behavior at sub-thermal energies.

The ENDF- 6 formatted thermal scattering sublibrary for the revised $\mathrm{Y}-\mathrm{YH}_{2} \mathrm{TSL}$ was then processed by the NDEX nuclear data processing code [17]. The total scattering cross sections for $\mathrm{Y}^{-} \mathrm{YH}_{2}$ as a function of 
temperature are provided for both the new (solid lines) and ENDF/B-VIII.0 (dashed lines) evaluations calculated by NDEX in Fig. 5. The effects of coherent elastic scattering on the $\mathrm{Y}^{-} \mathrm{YH}_{2}$ scattering cross section are clearly illustrated. Bragg peaks associated with the $\mathrm{YH}_{2} \mathrm{FCC}$ crystal structure and the expected temperature dependence on inelastic cross sections below the Bragg cutoff are present in the new $\mathrm{Y}_{-} \mathrm{YH}_{2}$ evaluation. The ENDF/B-VIII.0 evaluation significantly overestimates the scattering cross section below the Bragg cutoff.
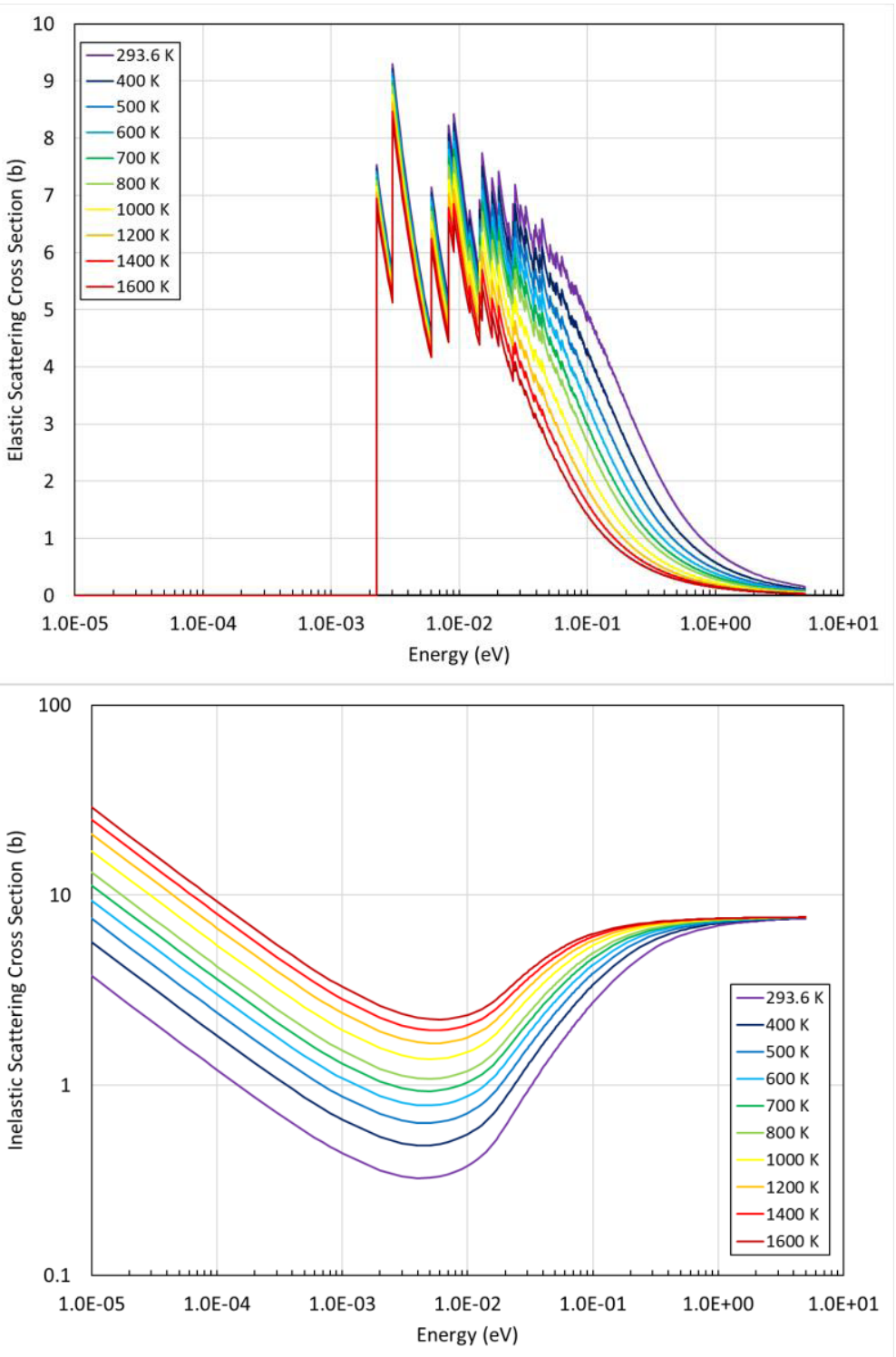

Figure 4. $\mathrm{Y}-\mathrm{YH}_{2}$ Coherent Elastic (top) and Incoherent Inelastic (bottom) Scattering Cross Sections

\subsection{Validation}

Validation of the $\mathrm{Y}-\mathrm{YH}_{2}$ TSL will be challenging because thermal neutron scattering in $\mathrm{YH}_{2}$ is dominated by hydrogen scattering effects. Table I compares the bound coherent $\left(\sigma_{\text {coh }}\right)$, bound incoherent $\left(\sigma_{\text {inc }}\right)$, total bound scattering $\left(\sigma_{\mathrm{b}}\right)$, and $2200 \mathrm{~m} / \mathrm{s}$ absorption cross sections for ${ }^{1} \mathrm{H}$ and ${ }^{89} \mathrm{Y}$ from the NIST neutron scattering database [18]. Hydrogen provides about $96 \%$ and yttrium $4 \%$ of the contribution to the total bound scattering cross section for $\mathrm{YH}_{2}$. 


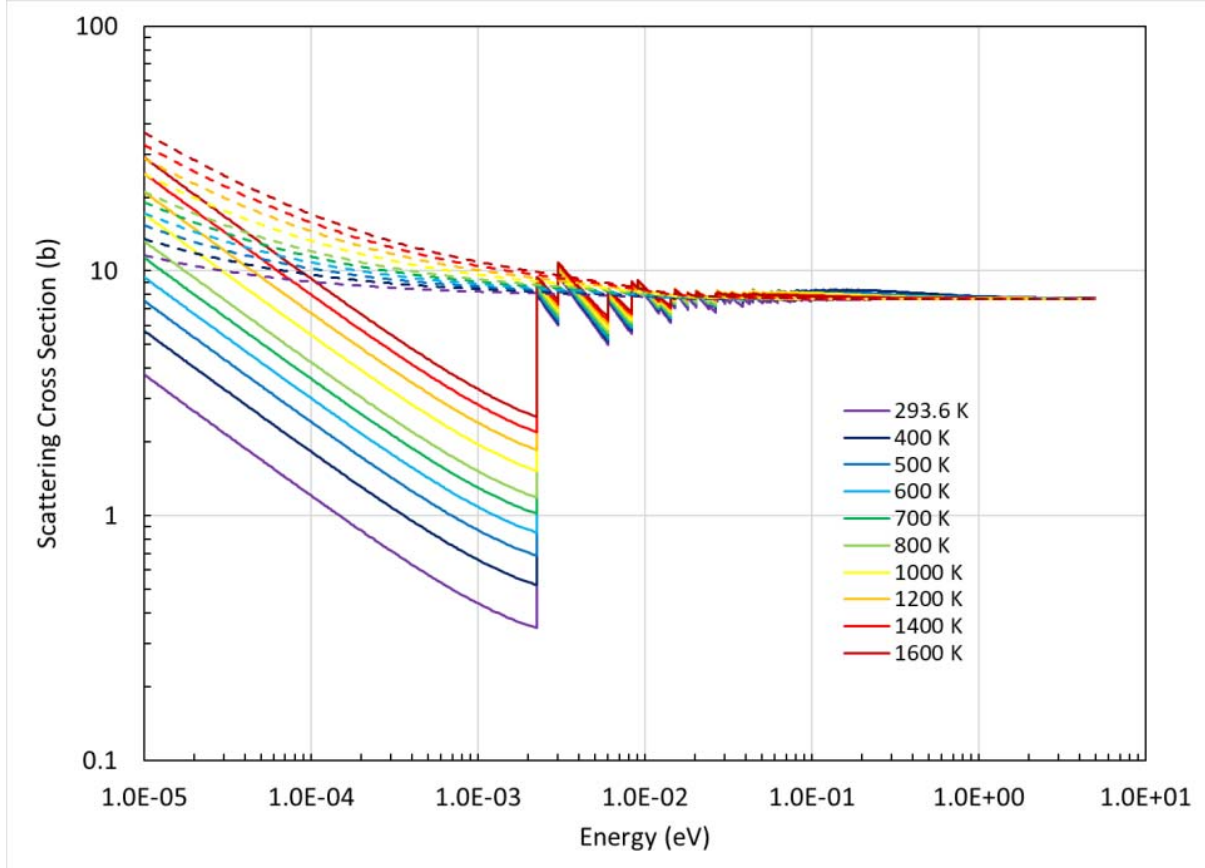

Figure 5. Total Scattering Cross Section for New (solid) and ENDF/B-VIII.0 (dashed) $\mathrm{Y}_{-} \mathrm{YH}_{2}$ Evaluations Calculated by NDEX

Table I. Bound Scattering Cross Sections [18]

\begin{tabular}{|c|c|c|c|c|c|}
\hline Nuclide & $\begin{array}{c}\text { Atomic } \\
\text { Mass Ratio }\end{array}$ & $\sigma_{\text {coh }}(\mathrm{b})$ & $\sigma_{\text {inc }}(\mathrm{b})$ & $\sigma_{\mathrm{b}}(\mathrm{b})$ & $\sigma_{\mathrm{a}}(\mathrm{b})$ \\
\hline${ }^{1} \mathrm{H}$ & 0.9991673 & $1.7583(10)$ & $80.27(6)$ & $82.03(6)$ & $0.3326(7)$ \\
\hline${ }^{89} \mathrm{Y}$ & 88.142104 & $7.55(4)$ & $0.15(8)$ & $7.70(9)$ & $1.28(2)$ \\
\hline
\end{tabular}

Prior neutron transmission measurement of $\mathrm{YH}_{x}$ compositions for $x \sim 2.0$ have concentrated on measurement of the $\mathrm{H}$ scattering since it dominates and have experimentally removed the contribution from $\mathrm{Y}[19,20]$. These measurements also had insufficient accuracy to resolve the Bragg edges in $\mathrm{YH}_{x}$ and could not measure neutron energies below the Bragg cutoff.

However, it should be possible to experimentally resolve coherent scattering effects using a series of neutron transmission measurements of $\mathrm{YH}_{x}$ specimens with progressively increasing bulk hydrogen content between $0.25<x \leq 2.0$. Table II provides a comparison of the contributions to the total bound scattering cross section of $\mathrm{YH}_{x}$ from $\mathrm{Y}$ and $\mathrm{H}$ scattering, normalized on a unit $\mathrm{Y}$ atom basis, for several $\mathrm{YH}_{x}$ compositions. The $\mathrm{Y}$ contribution to the total bound scattering cross section appears to be sufficient to permit the detection of coherent scattering effects for compositions with $x \leq 1.0$. These experiments will need to be limited to $x>0.25$ due to the usually high solubility limit of $\mathrm{H}$ in $\alpha-\mathrm{Y}$ [21]. Nevertheless, this threshold is expected to result in a $\mathrm{H}$ contribution to the cross section that is sufficiently low to distinguish the Bragg effects. For $0.25<x<1.86, \mathrm{YH}_{2}$ forms a two-phase solid solution with $\alpha$-Y metal [21]. Consequently, a TSL for $\alpha-Y$, which has a hexagonal close packed (HCP) crystal structure, will need to be 
developed in order to distinguish $\mathrm{Y}$ coherent scattering effects from $\mathrm{FCC} \mathrm{YH}_{2}$ and $\mathrm{HCP} \alpha-\mathrm{Y}$ in the $\mathrm{YH}_{x}$ samples.

A sub-thermal neutron production target is currently under development at the Rensselaer Polytechnic Institute (RPI) for use in low-energy neutron transmission measurements at the Gaerttner electron linear accelerator (LINAC). The sub-thermal neutron production target is being developed to perform neutron transmission measurements for moderator material and is expected to be able to support measurements with sufficient accuracy to detect Bragg peaks and the change in the total cross section below the Bragg cutoff for material with significant coherent scattering effects.

Table II. Comparison of Hydrogen and Yttrium Fractions in the Total Bound Scattering Cross Sections of Various Bulk YH $\mathrm{H}_{x}$ Compositions (Unit Y Atom Basis)

\begin{tabular}{|c|c|c|c|c|c|c|}
\hline & \multicolumn{2}{|c|}{} & \multicolumn{2}{c|}{$\begin{array}{c}\text { Traction of Total } \\
\text { Molal Bound Scattering } \\
\text { Cross Section (b) }\end{array}$} & \multicolumn{2}{c|}{$\begin{array}{c}\text { Bound Scattering } \\
\text { Cross Section }\end{array}$} \\
\cline { 2 - 7 }$x$ & $\alpha-\mathrm{Y}$ & $\mathrm{YH}_{2}$ & $\mathrm{Y}$ & $\mathrm{H}$ & $\mathrm{Y}$ & $\mathrm{H}$ \\
\hline 0.00 & 1.00 & 0.00 & 7.70 & 0.00 & 1.00 & 0.00 \\
\hline 0.25 & 0.88 & 0.13 & 7.70 & 20.51 & 0.27 & 0.73 \\
\hline 0.50 & 0.75 & 0.25 & 7.70 & 41.01 & 0.16 & 0.84 \\
\hline 0.75 & 0.63 & 0.38 & 7.70 & 61.52 & 0.11 & 0.89 \\
\hline 1.00 & 0.50 & 0.50 & 7.70 & 82.03 & 0.09 & 0.91 \\
\hline 1.25 & 0.38 & 0.63 & 7.70 & 102.54 & 0.07 & 0.93 \\
\hline 1.50 & 0.25 & 0.75 & 7.70 & 123.04 & 0.06 & 0.94 \\
\hline 1.60 & 0.20 & 0.80 & 7.70 & 131.25 & 0.06 & 0.94 \\
\hline 1.75 & 0.13 & 0.88 & 7.70 & 143.55 & 0.05 & 0.95 \\
\hline 1.86 & 0.07 & 0.93 & 7.70 & 152.57 & 0.05 & 0.95 \\
\hline 2.00 & 0.00 & 1.00 & 7.70 & 164.06 & 0.04 & 0.96 \\
\hline
\end{tabular}

\section{CONCLUSIONS}

The TSL for $\mathrm{Y}_{-} \mathrm{YH}_{2}$ has been re-evaluated using the FLASSH generalized elastic scattering capability in order to account for yttrium coherent elastic scattering effects which were neglected in the prior ENDF/BVIII.0 evaluation due to limitations in the LEAPR module of NJOY. The elastic scattering cross sections were calculated in the cubic approximation using the FCC crystal structure and room temperature lattice parameter of $\mathrm{YH}_{2}$. The resulting elastic scattering cross sections contain Bragg peaks consistent with the $\mathrm{YH}_{2}$ crystal structure. The inelastic scattering cross sections were calculated in the incoherent approximation using the same calculated Y phonon DOS used to prepare the ENDF/B-VIII.0 Y-YH 2 evaluation and a phonon expansion order of 300. The ENDF-6 File 7 produced by FLASSH was processed by NDEX and the resulting total scattering cross sections for $\mathrm{Y}^{-} \mathrm{YH}_{2}$ exhibit the expected Bragg peaks and cutoff for $\mathrm{YH}_{2}$.

Since thermal neutron scattering in $\mathrm{YH}_{2}$ is dominated by scattering from hydrogen, experimental validation of the $\mathrm{Y}-\mathrm{YH}_{2}$ TSL will be challenging. An experimental approach to validate the $\mathrm{Y}^{-} \mathrm{YH}_{2}$ TSL using neutron transmission measurement of $\mathrm{YH}_{x}$ samples is discussed. The approach is enabled by the sub-thermal target currently under development at the RPI LINAC. 


\section{REFERENCES}

1. W. M. Mueller, J. P. Blackledge and G. G. Libowitz, Metal Hydrides, pp. 443-449, Academic Press, New York, USA (1968).

2. A. I. Hawari, "Modern Techniques for Inelastic Neutron Scattering Analysis," Nuclear Data Sheets, 118, pp. 172-175 (2014).

3. R. E. MacFarlane, "New Thermal Neutron Scattering Files for ENDF/B-VI Release 2," LA-12639-MS, Los Alamos National Laboratory (1994).

4. D. A. Brown et al., "ENDF/B-VIII.0: The $8^{\text {th }}$ Major Release of the Nuclear Reaction Data Library with CIELO-project Cross Sections, New Standards and Thermal Scattering Data," Nuclear Data Sheets, 148, pp. 1-142 (2018).

5. M. Zerkle and J. Holmes, "A Thermal Neutron Scattering Law for Yttrium Hydride," EPJ Web of Conferences, 146, 13005 (2017).

6. A. C. Kahler, "The NJOY Nuclear Data Processing System, Version 2012," LA-UR-12-27079, Los Alamos National Laboratory (2012).

7. Y. Zhu and A. I. Hawari, "Full Law Analysis Scattering System Hub (FLASSH)," Proceedings of PHYSOR2018, Cancun, Mexico, April 22-26 (2018).

8. Y. Zhu and A. I. Hawari, "Implementation of a Generalized Coherent Elastic Scattering Formulation for Thermal Neutron Scattering Analysis, Proceedings of International Conference on Nuclear Criticality Safety, Charlotte, North Carolina, September 13-17 (2015).

9. G. Kresse and J. Furthmüller, "Efficient Iterative Schemes for Ab Initio Total-Energy Calculations Using a Plane-Wave Basis Set," Physical Review B, 54(16), pp. 11169-17979 (1996).

10. G. Kresse and J. Furthmüller, "Efficiency of Ab-Initio Total Energy Calculations for Metals and Semiconductors using a Plane-wave Basis Set," Computational Materials Science, 6, pp. 15-50 (1996).

11. J. P. Perdew, K. Burke and M. Ernzerhof, "Generalized Gradient Approximation Made Simple," Physical Review Letters, 77(18), pp. 3865-3868 (1996).

12. J. N. Daou and P. Vajda, "Hydrogen Ordering and Metal-Semiconductor Transitions in the System $\mathrm{YH}_{2+x}$, " Physical Review B, 45, pp. 10907-10912 (1992).

13. K. Parlinski, Z. Q. Li and Y. Kawazoe, "First-Principles Determination of the Soft Mode in Cubic $\mathrm{ZrO}_{2}$," Physical Review Letters, 78(21), pp. 4063-4066 (1997).

14. H. E. Flotow, D. R. Osborne and K. Otto, "Heat Capacity and Thermodynamic Functions of $\mathrm{YH}_{2}$ and $\mathrm{YD}_{2}$ from $5^{\circ}$ to $350^{\circ} \mathrm{K}$ and the Hydrogen Vibration Frequencies," Journal of Chemical Physics, 36, pp. 866-872 (1962).

15. J. J. Rush, H. E. Flotow, D. W. Connor and C. L. Thaper, "Vibration Spectra of Yttrium and Uranium Hydrides by the Inelastic Scattering of Cold Neutrons, Journal of Chemical Physics, 45(10), pp. 38173825 (1966).

16. T. J. Udovic, J. J. Rush and I. S. Anderson, "Local-Mode Dynamics in $\mathrm{YH}_{2}$ and $\mathrm{YD}_{2}$ by Isotope-Dilution Neutron Spectroscopy," Physical Review B, 50(21), pp. 15739-15743 (1994).

17. T. H. Trumbull, "Computational Methods used to Process Thermal Neutron Scattering Data for use in Continuous Energy Monte Carlo Codes," Proceeding of PHYSOR2016, Sun Valley, Idaho, May 1-5 (2016).

18. V. F. Sears, "Neutron Scattering Lengths and Cross Sections," Neutron News, 3(3), pp. 26-37 (1992).

19. P. Vorderwisch and K. Waßerroth, "Total Neutron-Proton Scattering Cross Section of Yttrium Hydride," Atomkernenergie, 14, 370 (1969).

20. K. Brand, "Total Neutron Cross Sections of Chemical Bound Protons and Deuterons in Some Metal Hydrides and Deuterides," Atomkernenergie, 17, pp. 113-120 (1971).

21. D. Khatamian and F. D. Manchester, "The H-Y (Hydrogen-Yttrium) system," Bulletin of Alloy Phase Diagrams, 9, pp. 252-260 (1988). 